\title{
Malignant epitheloid angiomyolipoma of the kidney in a child treated with sunitinib, everolimus and axitinib
}

\author{
Elvan Caglar Citak, MD; ${ }^{*}$ Eda Bengi Yilmaz, MD; ${ }^{\dagger}$ Emel Yaman, MD, ${ }^{\ddagger}$ Simge Kaya, MD; ${ }^{*}$ Hakan Taskinlar, MD;* \\ Rabia Bozdogan Arpaci, MD; Demir Apaydin, MD
}

*Department of Pediatric Oncology, Mersin University, Turkey; ${ }^{\dagger}$ Department of Radiation Oncology, Mersin University, Turkey; ${ }^{\circledR}$ Department of Medical Oncology, Mersin University, Turkey; ${ }^{*}$ Department of Pathology, Mersin University, Turkey; „Department of Pediatric Surgery, Mersin University, Turkey; £Department of Radiology, Mersin University, Turkey

Cite as: Can Urol Assoc J 2015;9(7-8):E542-5. http://dx.doi.org/10.5489/cuaj.2823 Published online July 17, 2015.

\section{Abstract}

The malignant variant of epithelioid angiomyolipoma (EAML) of the kidney is uncommon, extremely aggressive and behaves like a renal cell carcinoma. We present a case of a 12-year-old male with malignant EAML who was treated according to adult treatment protocols. To our knowledge, axitinib has not been used before in children. We conclude that adult protocols, in this rare case, could be safely used in rare childhood malignancies.

$\mathrm{E}$ pithelioid angiomyolipoma (EAML) of the kidney is rare and strongly associated with tuberous sclerosis. Its malignant variant is uncommon, extremely aggressive, ${ }^{1}$ and behaves like a renal cell carcinoma (RCC). Targeted therapies may offer some hope in cases of progressive malignant EAML when surgery is not amenable. ${ }^{2}$ We present a 12-year-old male with malignant EAML who did not respond to approved therapies.

\section{Case report}

A 12-year-old male presented to the outpatient department complaining of spots on his face. A physical examination revealed fibromas and hypo- and hyperpigmented macules spread over the body. An abdominal computed tomography (CT) revealed bilateral angiomyolipoma (Fig. 1, part A), while nodular lesions around the lateral ventricles were identified in cranial magnetic resonance imaging and an ophthalmological examination revealed a left retinal astrocytoma. The patient was diagnosed with tuberosclerosis.

One year later the patient was admitted with abdominal pain and a mass on the left side of the abdomen. Abdominopelvic $\mathrm{CT}$ revealed a giant mass originating from the left kidney (Fig. 1, part B), for which a radical nephrectomy was performed. The pathologic specimens were evalu- ated at our pathology department and the Johns Hopkins Pathology Department, and were reported as malignant EAML with pleomorphism, necrosis, and atypical mitotic figures (Fig. 2, parts A, B). The tumour was labelled for cathepsin $\mathrm{K}$ and melan $\mathrm{A}$ and focally for HMB45, while rare cells were labelled for EMA, PAX8, Cam5.2 and no cells labelled for AE1/3 (Fig. 2, parts C, D).

A fluorescence in situ hybridization (FISH) was performed, although TFE3 and TFEB showed no evidence of such gene rearrangements. Treatment was started with sunitinib maleat. One month later, $\mathrm{CT}$ revealed the regression of the tumour (Fig. 1, part C); however, the patient was readmitted to hospital with complaints of a cough. A physical examination revealed tachypnea and decreased breathing sounds and a mass in the lower left of the abdomen. In the thorax CT, pleural effusion was noted (Fig. 1, part D) and cytological analysis revealed a metastatic epithelial tumour. Abdominopelvic CT showed progression of the tumour (Fig. 1, part E). Treatment was changed to everolimus after the effusion disappeared, and the patient underwent a consultation with a radiation oncologist, but radiotherapy was not indicated. After 3 months, CT revealed almost complete remission (Fig. 1, part F), but after 8 months of treatment, the patient was admitted with abdominal pain and a high fever. An evaluation CT again showed a progression of the tumour and a debulking surgery was performed. Treatment was changed to axitinib. In the second month of treatment, the patient underwent a CT which revealed no change in tumour size and showed metastasis of the thorax (Fig. 1, part $\mathrm{G}, \mathrm{H}$ ).

After 4 months of treatment, the patient was admitted to the emergency department with dyspnea. A CT revealed not only a giant mass, but also bilateral metastasis of the hemithorax and left pleural effusion. The patient was admitted to the intensive care unit. After 3 days of hospitalization, the patient underwent a mechanical ventilation. The patient died after 16 days of hospitalization, despite all supportive treatments. 


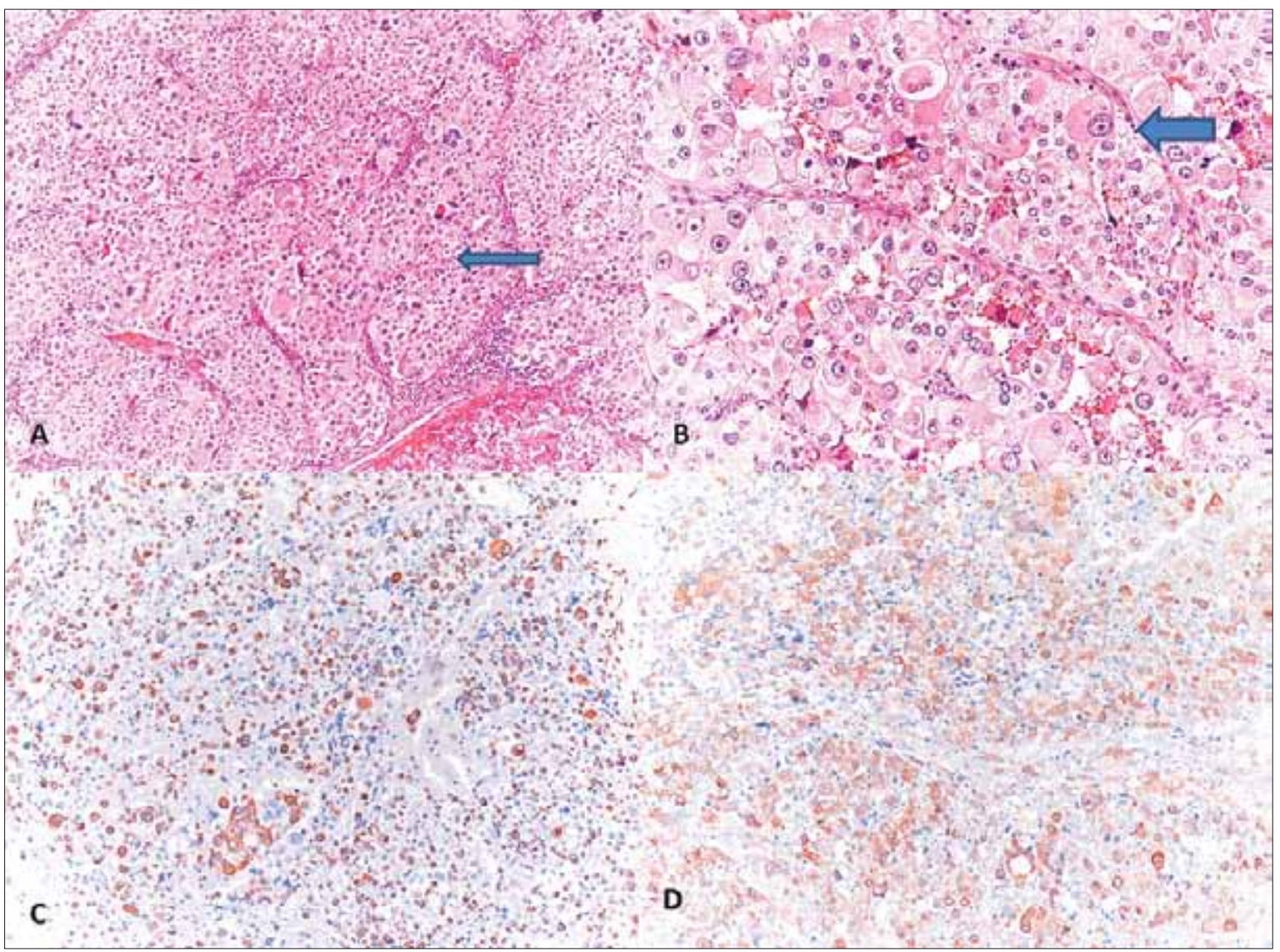

Fig. 1. (A): Bilateral renal lesions evaluated as angiomyolipomas; (B): A giant mass originating from the left kidney and occupying the left abdominal cavity; (C) Residual giant mass occupying the left part of the abdomen; (D): Pleural effusion occupying the left hemithorax; (E): Regression in the size of the giant mass in the left abdominal cavity; (F): Progression in the size of the residual tumour; (G): Giant residual tumor; (H): Massive left-sided and minimal right-sided pleural effusion.

\section{Discussion}

EAML is more aggressive than typical angiomyolipoma, with the capability of recurrence and metastasis. The case in point presented with a giant mass, necrosis, disease progression, and a carcinoma-like histology. Due to the univariate analysis reported in the study by Jayaprakash and colleagues, our case is considered as high risk. ${ }^{3}$ The time of metastasis during the follow-up was 17 to 31 months (median: 12-18 months). In this case, metastasis was seen in the third month of treatment. Given the presence of malignant behaviour, we decided to treat our case as RCC.

According to National Comprehensive Cancer Network (NCCN) guidelines, a radical nephrectomy is the only option in stage III tumours, ${ }^{4}$ but in our case, other treatment modalities were applied due to the residual tumour. Despite the recommendations of the NCCN panel, due to availability problems with sunitinib, a receptor tyrosine kinase inhibitor (RTK) was used instead of an inhibitor of the mammalian target of rapamycin (mTOR) inhibitors (e.g., everolimus, temsirolimus). The phase II data of 31 patients with non-clear cell RCC, who were treated with sunitinib, reported an overall response rate of $36 \%$ and a median progression-free survival of 6.4 months. ${ }^{5}$ Sunitinib is category $2 \mathrm{~A}$ recommendation for treatment-naive patients with stage IV non-clear cell RCC. ${ }^{4}$

The evaluation CT revealed the regression of the tumour under sunitinib treatment, but 2 months later, progression was observed, leading to treatment with everolimus. Data on the benefit of everolimus in patients with non-clear cell RCC are limited, although results from subgroup analyses of an expanded-access trial and case reports support the clinical use of everolimus in patients with non-clear cell RCC. ${ }^{6,7}$ In a phase III trial of everolimus by Bissler and colleagues, a significant advantage was reported for everolimus over placebo in reducing angiomyolipoma volume with an acceptable safety profile in patients with tuberous sclerosis. ${ }^{8}$ Everolimus was used due to problems in providing temsirolimus, and there was a significant response to everolimus in our patient; a CT 5 months later revealed complete remission. After the eighth month of therapy, due to progression, we decided to start treatment with axitinib. 


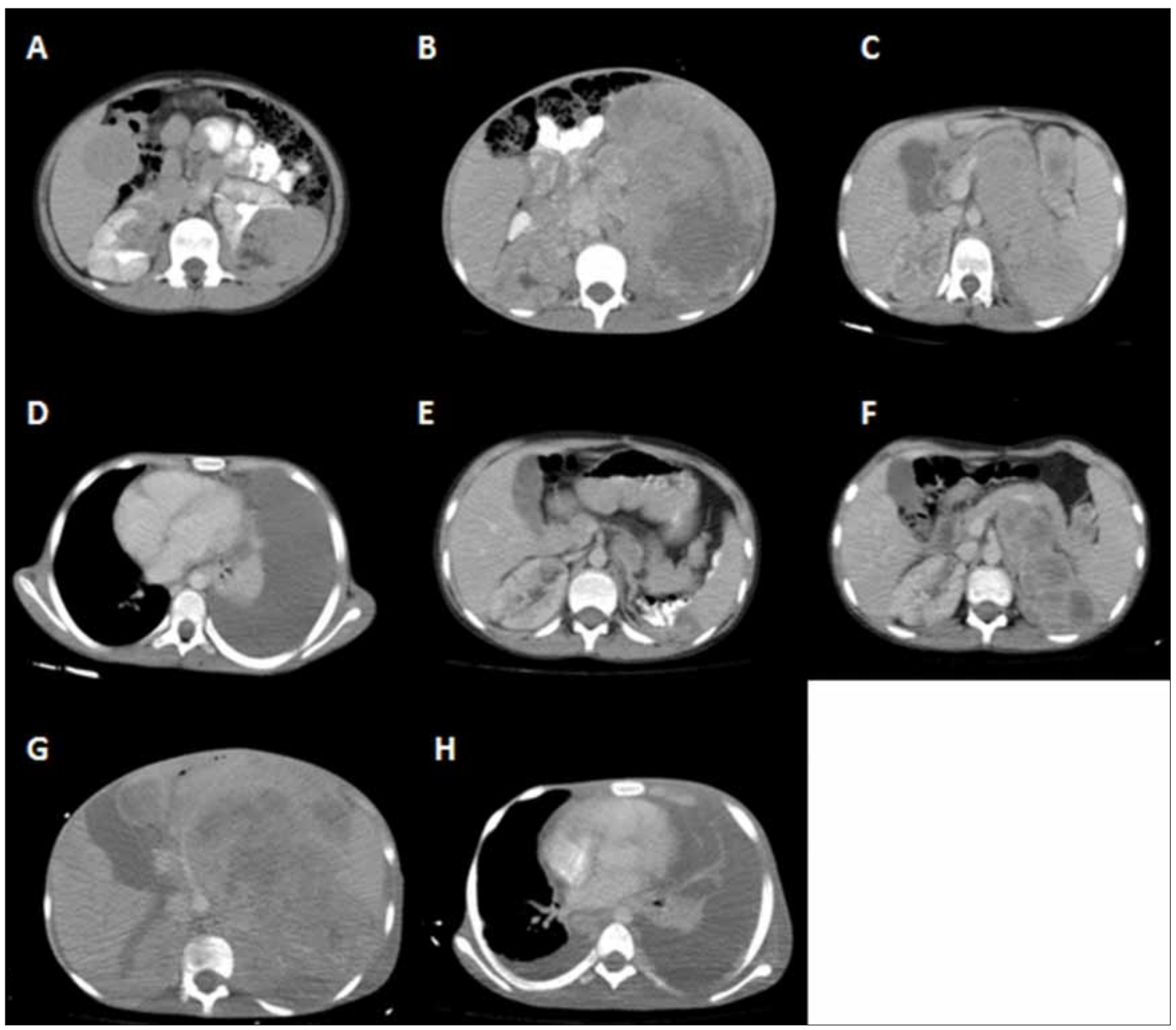

Fig .2. (A): Epithelioid-polygonal cells, and varying degrees of nuclear atypia (arrow) (hematoxylin and eosin stain [H\&E], $\times 100) ;(B)$ : Sheets of epitheloid cells with moderate amounts of eosinophilic cytoplasm and vesicular nucleus with prominent nucleoli (arrow) (H\&E, $\times 200$ ); (C): The tumour cells showed positive reactivity for Melan-A (Melan A, ×100); (D): The tumour cells showed positive reactivity for HMB-45 (HMB-45, $\times 100)$.

Axitinib is an active selective inhibitor of VEGFR 1, 2 and 3 that has been shown to reduce vascular permeability, tumour vascularization and tumour volume, including RCC. ${ }^{9}$ There are ongoing clinical trials evaluating the efficacy of axitinib in patients with non-clear cell RCC in the first- and second-line settings. ${ }^{9}$ Based on extrapolation, the NCCN Kidney Cancer Panel has included this therapy as first-line for patients with relapsed or medically unresectable stage IV disease with non-clear cell histology (category $2 \mathrm{~A}) .^{4}$

\section{Conclusion}

Our case is significant given that the child was treated according to adult protocols. We believe that the use of new and distinctive chemotherapeutics is necessary in cases of rare tumours that behave like RCC.
Acknowledgment: The authors thank Dr. Pedram Argani, the Johns Hopkins Medical Institutions, Baltimore, for evaluating the pathologic specimen and for the fluorescence in situ hybridization (FISH) analysis.

Competing interests: The authors declare no competing financial or personal interests.

This paper has been peer-reviewed.

\section{References}

1. Martignoni G, Amin MB. Angiomyolipoma. In: Eble JN, Sauter G, Epstein Jl, Sesterhenn IA, eds. Pathology and Genetic Tumours of the Urinary System and Male Genital Organs. Lyon, France: IARC; 2004:65-7.

2. Wyluda $E$, Baquero $G$, Lamparella $N$, et al. Fatal malignant metastastic epithelioid angiomyolipoma presenting in a young woman: Case report and review of the literature. Rare Tumors 2013:5:46. http:// dx.doi.org/10.4081/rt.2013.e46 
3. Jayaprakash PG, Mathews $S$, Azariah MB, et al. Pure epitheliod perivascular epitheloid cell tumor (epitheliod angiomyolipoma) of kidney: Case report and literature review. J Cancer Res Ther 2014:10:404-6. http:// dx.doi.org/10.4103/0973-1482.136672

4. National Comprehensive Cancer Network Clinical Practice Guidelines in Oncology Kidney Cancer Version 1.2015. http://www.nccn.org/professionals/physician_gls/pdf/kidney.pdf.

5. Lee IL, Ahn JH, Lim HY, et al. Multicenter phase II study of sunitinib in patients with non-clear cell renal cell carcinoma. Ann Oncol 2012;23:2108-14. http://dx.doi.org/10.1093/annonc/mdr586

6. Blank CU, Bono P, Larkin JMG, et al. and on behalf of the REACT Study Group. Safety and efficacy of everolimus in patients with non-clear cell renal cell carcinoma refractory to VEGF-targeted therapy: Subgroup analysis of REACT. J Clin Oncol 2012:402:30.

7. Koh Y, Kim JY, Lim HY, et al. Phase II trial of RAD 001 in renal cell carcinoma patients with non-clear cell histology. J Clin Oncol 2012:30:4544.
8. Bissler JJ, Kingswood JC, Radzikowska E, et al. Everolimus for angiomyolipoma associated with tuberous sclerosis complex or sporadic lymphangioleiomyomatosis (EXIST-2): A multicentre, randomised, doubleblind, placebo-controlled trial. Lancet 2013;381:817-24.

9. Rini B, Rixe 0, Bukowski R, et al. «AG-013736, a multi-target tyrosine kinase receptor inhibitor, demonstrates anti-tumor activity in a Phase 2 study of cytokine-refractory, metastatic renal cell cancer (RCC)». J Clin Oncol ASCO Annual Meeting Proceedings 2005;23:4509.

Correspondence: Dr. Elvan Caglar Citak, Department of Pediatric Oncology, Mersin University, Turkey; caglarcitak@yahoo.com 